\title{
Loss of neuropathy target esterase in mice links organophosphate exposure to hyperactivity
}

\author{
Christopher J. Winrow ${ }^{1,3}$, Matthew L. Hemming ${ }^{1}$, Duane M. Allen ${ }^{1}$, Gary B. Quistad ${ }^{2}$, John E. Casida ${ }^{2}$ \\ \& Carrolee Barlow ${ }^{1,3}$
}

Published online 17 March 2003; doi:10.1038/ng1131

\begin{abstract}
Neuropathy target esterase (NTE) is involved in neural development and is the target for neurodegeneration induced by selected organophosphorus pesticides and chemical warfare agents. We generated mice with disruptions in Nte, the gene encoding NTE. Nte ${ }^{-/-}$mice die after embryonic day 8 , and $\mathrm{Nte}^{+-}$mice have lower activity of Nte in the brain and higher mortality when exposed to the Nte-inhibiting compound ethyl octylphosphonofluoridate (EOPF) than do wild-type mice. $\mathrm{Nte}^{+-}$and wild-type mice treated with $1 \mathrm{mg}$ per $\mathrm{kg}$ of body weight of EOPF have elevated motor activity, showing that even minor reduction of Nte activity leads to hyperactivity. These studies show that genetic or chemical reduction of Nte activity results in a neurological phenotype of hyperactivity in mammals and indicate that EOPF toxicity occurs directly through inhibition of Nte without the requirement for Nte gain of function or aging.
\end{abstract}

\section{Introduction}

The acute toxic effects of organophosphate insecticides and chemical warfare agents are due to inhibition of acetylcholinesterase $e^{1,2}$. There are also delayed effects for a subset of organophosphates. In humans, delayed chronic neurotoxic syndromes from exposure to individual organophosphates or combinations of agents have been reported for Gulf War veterans and chronically exposed individuals $^{3-9}$. The most common and best understood delayed syndrome is organophosphate-induced delayed neuropathy (OPIDN). Current knowledge of OPIDN is based on studies of more than 30,000 human cases and the use of hens as the preferred model. OPIDN is characterized by paralysis of the lower limbs due to degeneration of long axons in the spinal cord and in peripheral nerves. The proposed target protein is NTE ${ }^{10,11}$.

The multistep hypothesis of organophosphate toxicity is as follows: (i) organophosphate toxicants selectively inhibit NTE relative to acetylcholinesterase; (ii) neuropathic effects are observed only after NTE activity is inhibited by $70-90 \%$ (refs. 11,12); (iii) NTE is phosphorylated at the serine residue in the catalytic site; (iv) loss of an alkoxy group (referred to as aging) leaves a negatively charged phosphate at the active site; (v) a toxic gain of function leads to neurodegeneration $^{10-13}$. But the organophosphates used to establish the multistep hypothesis may have multiple targets, and the association with NTE inhibition may be an epiphenomenon. Elucidation of the primary structure of human NTE and mouse Nte did not in itself suggest a physiologic function but did establish homology to the Drosophila melanogaster swiss cheese (SWS) protein ${ }^{14,15}$. SWS is not essential for embryonic survival, but flies lacking the sws gene undergo glial hyperwrapping and subsequent neurodegeneration followed by death ${ }^{16}$. These are the only known consequences of reducing levels of SWS and, by analogy, NTE in an organism. Therefore, it is important to define the role of NTE in mammals and the consequences of its inhibition by organophosphates.

Characterization of the mouse and human genes encoding NTE (Nte and NTE, respectively) is a key step in dissecting the function of NTE and its role in organophosphate-induced toxicities. The mouse is a useful model for studying the effects of organophosphates $^{17-19}$, even though it has a different spectrum of neuropathology and toxicity than does the hen ${ }^{11,12,20,21}$. Organophosphate-induced sub-acute neurotoxicity in mice is similar to OPIDN in hens in the correlation with NTE inhibition and the prophylactic actions of reversible NTE inhibitors but differs from OPIDN in the time of onset of toxicity and a greater incidence of fatality ${ }^{19,21,22}$.

Here we report the mapping and characterization of the NTE and Nte genomic loci and describe the generation of mice lacking Nte. Unlike sws in D. melanogaster, Nte is essential for embryonic survival. In addition, we found that mice heterozygous with respect to the Nte mutation $\left(\mathrm{Nte}^{+-}\right)$had lower Nte activity in the brain, greater motor activity and greater sensitivity to EOPF. Our study shows that it is the reduction of NTE activity and not a gain-offunction phenotype that is responsible for the toxic effects of organophosphate agents. This is the first system for studying the effects of reduced NTE levels in mammals and provides a muchneeded model system for carrying out analyses of potential clinical syndromes caused by organophosphate exposure.

\footnotetext{
${ }^{1}$ The Salk Institute for Biological Studies, The Laboratory of Genetics, 10010 North Torrey Pines Road, La Jolla, California 92037, USA. ${ }^{2}$ Environmental Chemistry and Toxicology Laboratory, Department of Environmental Science, Policy and Management, 115 Wellman Hall, University of California, Berkeley, California 94720-3112, USA. ${ }^{3}$ Present address: Merck Research Laboratories, 3535 General Atomics Court, San Diego, California 92121, USA.
} Correspondence should be addressed to C.B. (e-mail: carrolee_barlow@merck.com). 


\section{Results}

\section{Characterization of NTE and Nte genomic loci}

To study the Nte genomic locus, we sequenced a mouse expressedsequence tag (EST) clone homologous to the $5^{\prime}$ end of NTE and used it to design primers to screen a 129S6/SvEvTac (129S6) mouse bacterial artificial chromosome (BAC) library (Incyte Genomics, Down-to-the-Well platform). This screen identified BAC clone 59B13. We sequenced this clone throughout the Nte locus and used it as a probe for fluorescence in situ hybridization (FISH) of mouse metaphase chromosome spreads (Fig. 1a). We also identified a human BAC clone (48M5) and used it for FISH of human metaphase chromosomes (Fig. 1a). FISH showed that the NTE loci mapped to human chromosome 19p13.3 and mouse chromosome 8A1.1, consistent with data from the National Center for Biotechnology Information (NCBI). A high level of conservation is observed between the NTE and Nte genomic loci (Fig. 1b). Both loci span 35 exons, over $27 \mathrm{~kb}$ in human and $29 \mathrm{~kb}$ in mouse, and intron-exon boundaries are highly conserved (Fig. 1b). By combining standard library screening with database searches, we also identified an Nte liver splice variant (Fig. $1 b$ ). This alternate transcript contains 14 exons, is $1659 \mathrm{bp}$ in length, uses an exon not present in the predominant form of Nte and lacks the Nte esterase (NEST) domain and two of three cyclic nucleotide monophosphate (cNMP)-binding domains ${ }^{23}$.

$a$
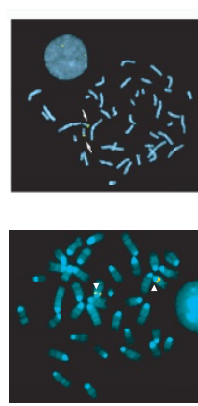

$b$
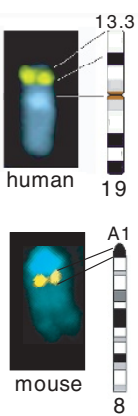
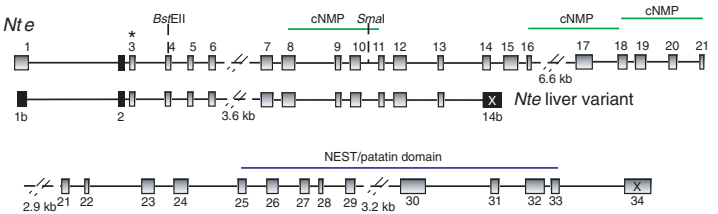

NTE

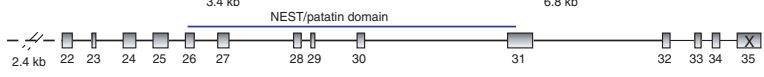

$1 \mathrm{~kb}$

C

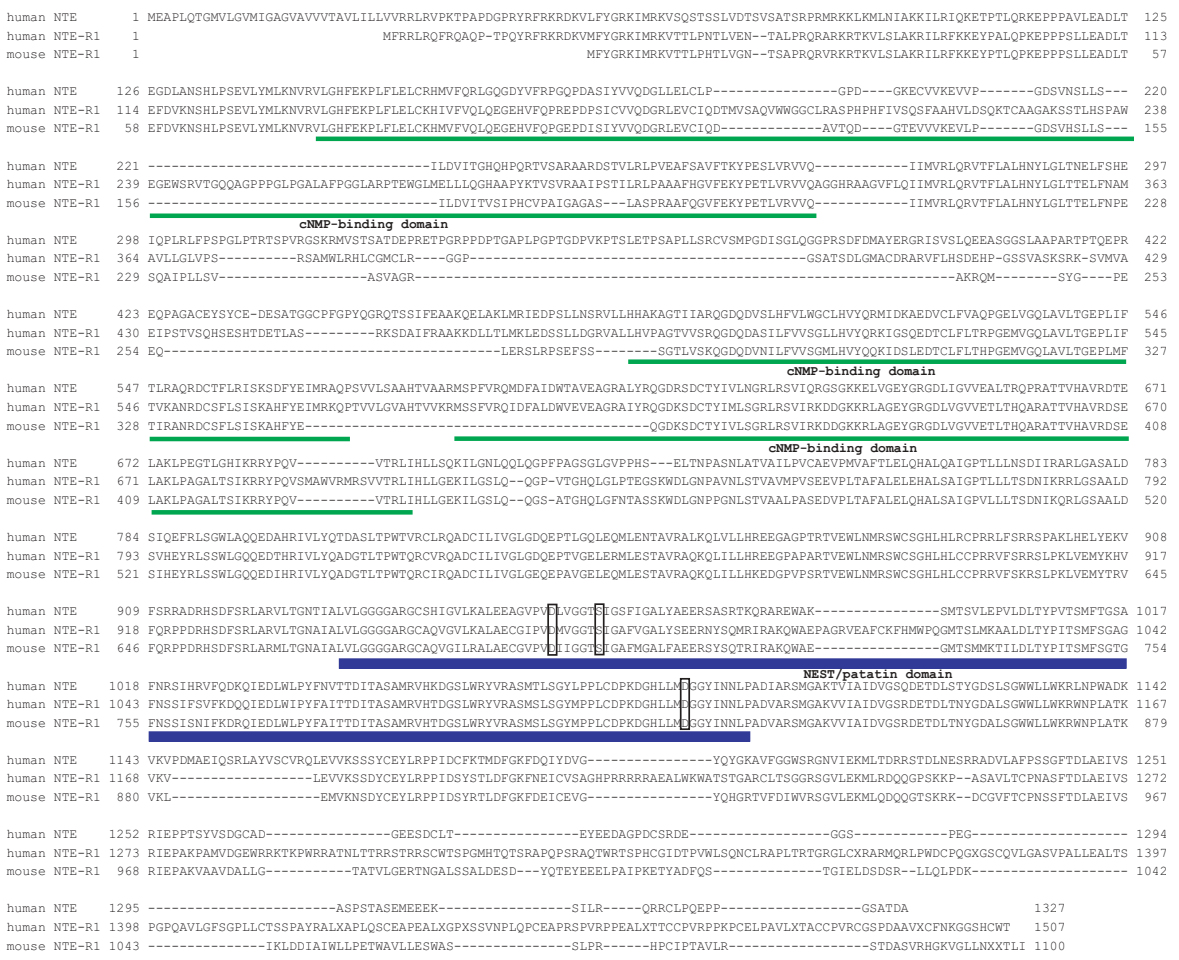

Identification of an NTE-related gene

In silico analysis of public and commercial databases also identified a related NTE locus termed NTE-related 1 (NTE-R1). We ntified partial clones in the GenBank database and the full genomic locus using the Celera Discovery System. The NTE-R1 locus is conserved in mouse, rat and human (Fig. 1c). The man NTE-R1 gene is localized to chromosome 9 and spans 31 some 2 and spans 27 exons over $74.7 \mathrm{~kb}$. We identified several STs matching the NTE-R1 transcript in human and mouse in multiple locations. The predicted transcript is $3305 \mathrm{bp}$ in length in mouse and $4522 \mathrm{bp}$ in humans. There is a high degree of $74 \%$ identity in mice) and amino-acid levels, with $65 \%$ homology (55\% identity) in humans and 73\% homology (62\% identhe catalytic residues) is retained in NTE-R1 (Fig. 1c) and is highly conserved with $82 \%$ homology (68\% identity) in human

\section{Generation of mice deficient in Nte}

We generated a restriction map of the 129S6 BAC clone 59B13 and used a genomic fragment of $15.2 \mathrm{~kb}$ to construct the Nte targeting vector (Fig. 2a). The targeting vector was designed to result in an in-frame fusion between exon 4 of Nte and the lacZ gene (lacking a start ATG and promoter) and to delete exons 5-10 by inserting the neomycin-resistance gene $\left(n e o^{r}\right)$ followed by multiple stop codons. With correct recombination a full-length

Fig. 1 Characterization of the NTE and Nte genomic loci and identification of NTE-R1. a, FISH analysis was done on human metaphase chromosomes (upper panel) using the BAC clone $48 \mathrm{M} 5$ and on mouse metaphase chromosomes (lower panel) using the BAC clone 59B13. NTE is localized to chromosome 19p13.3 and Nte to chromosome 8A1.1. $\boldsymbol{b}$, Schematic of the NTE loci in mouse (top) and human (bottom) and the alternatively spliced Nte transcript isolated from liver (NTE liver variant). Exons are shown as gray blocks, and those containing the stop codon are indicated with an " $X$ ". The exon containing the start ATG is indicated by an asterisk and solid black blocks indicate alternatively spliced exons found in the Nte liver variant. The conserved cNMP-binding domains and the NEST/patatin domain are indicated by green and blue lines, respectively. The BstEII and Smal sites used for constructing the Nte targeting vector are shown. The schematic also shows the high level of intron/exon conservation between the NTE and Nte loci. c, Clustal alignment of human NTE and the human NTE-R1 and mouse Nte-R1 proteins. The three cNMP-binding domains (green line) and the NEST/patatin domain (blue line) are conserved among all three proteins. Amino acids that form the catalytic triad within the NEST/patatin domain are also conserved and are shown in boxes. 
Fig. 2 Generation of mice with disruptions in Nte. a, The Nte-lacZ targeting vector is shown above the normal $\mathrm{Nte} \mathrm{H}^{++}$locus and the targeted locus. Exons are indicated as gray blocks with exon numbers given below the targeted locus. $\beta$-galactosidase (Glb1) neomycin resistance $\left(n e o^{r}\right)$ and thymidine kinase (HSV-TK) genes are shown and a probe used for Southern-blot screening is indicated as a horizontal bar. Correct integration results in the elimination of BstEll and Smal sites flanking the genes Glb1 and neor (indicated by strikethroughs). $\boldsymbol{b}$, The correctly targeted locus results in the in-frame fusion of the lacZ gene to Nte exon 4 at a BstEll site deletion of exons 5-10 and insertion of the neor gene at a Smal site. The lac $Z$ and $n e o^{r}$ genes contain stop codons and polyA sites, and the downstream exons are out of frame from exons 1 and 2 . thereby decreasing the likelihood of splicing around the targeted insertion. $c$, Southern blot of genomic DNA from F1 mice, digested with BstEll and hybridized with the probe shown in a, showing correct integration of the disrupted $\mathrm{Nte}$ allele in the $\mathrm{Nte}^{+/-}$mice (lanes with an asterisk).

Nte transcript would not be produced. Expression of lacZ from the endogenous Nte promoter (Fig. 2b) allowed for the visualization of $\beta$-galactosidase activity in the mutant mice, corresponding to the normal pattern of Nte expression. The overall result of this strategy was the elimination of a functional Nte transcript.

We isolated a set of 114 neomycin-resistant embryonic stem cell (ES) colonies. Nine ES clones were correctly targeted and four independent ES clones were used to generate chimeras. Two of the chimeras from independent ES clones showed high-level germline transmission. We mated the chimeras to obtain offspring carrying the mutated allele. Southern-blot analysis of tail samples identified mice with a properly targeted Nte allele

$a$

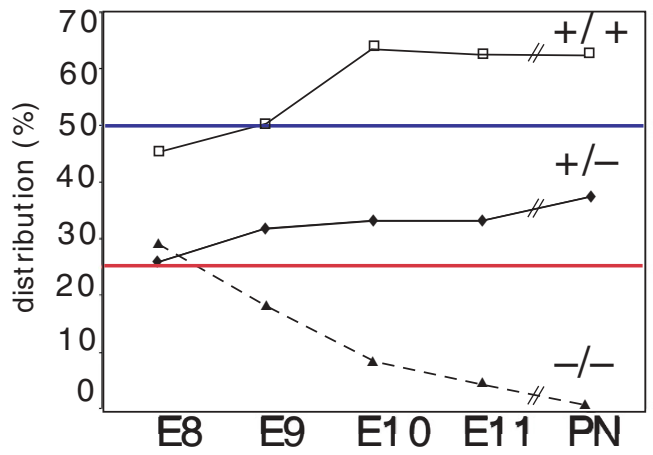

$b$

E11
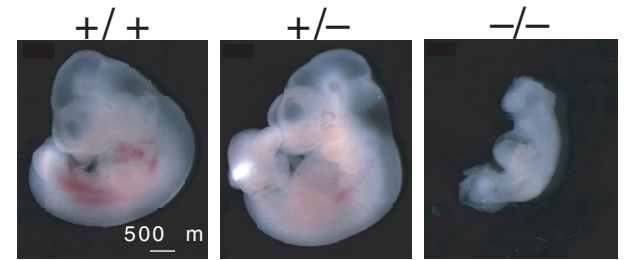

E10
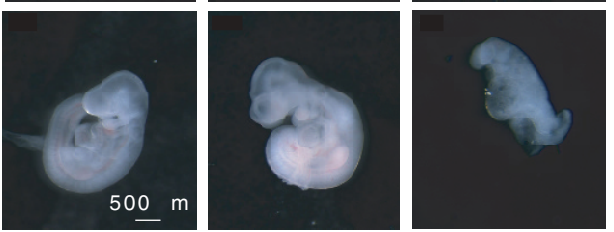

E9
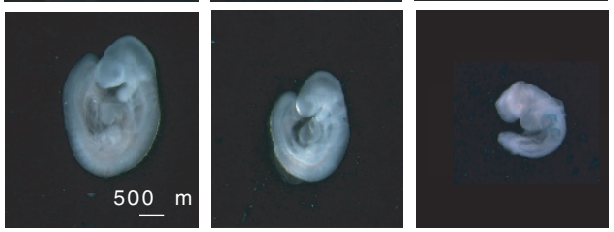

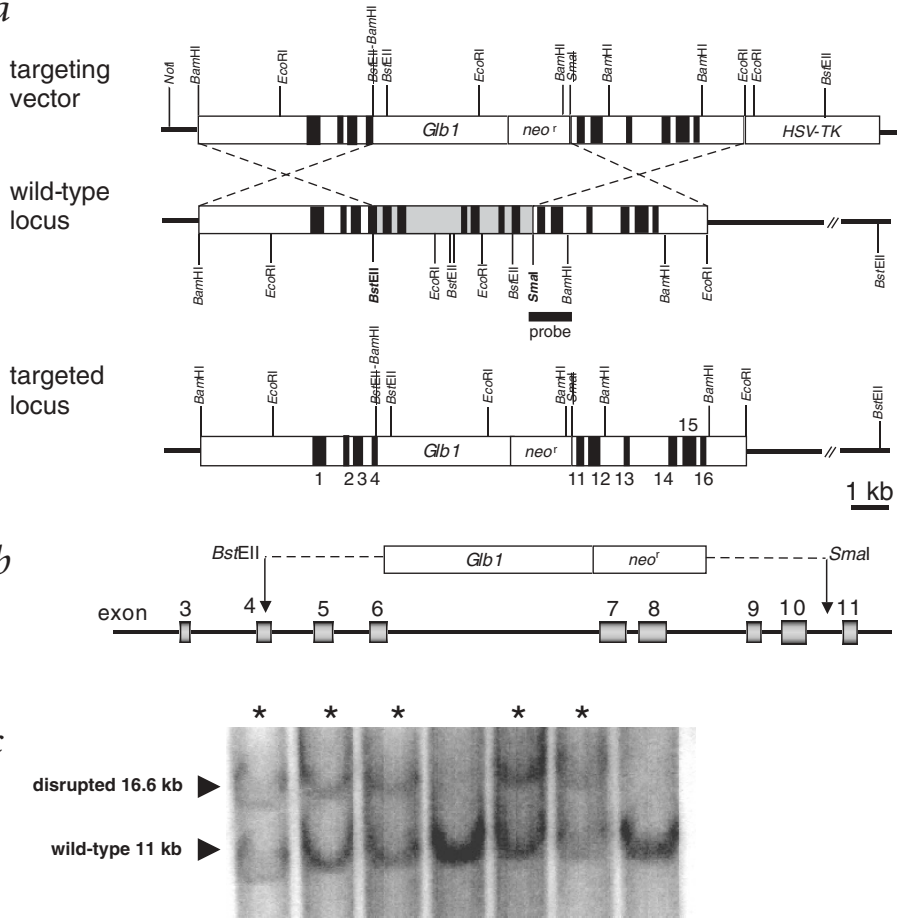

(Fig. 2c). Both independent ES clones gave rise to mouse lines with identical phenotypes.

\section{Nte is essential for embryonic survival}

Northern-blot analysis showed that Nte was expressed as early as embryonic day 7 (E7) and throughout embryonic development (data not shown). Loss of both Nte alleles resulted in lethality evident at E9 (Fig. 3). The expected genotype distribution of mutant, wild-type and heterozygous mice was observed at E8 (Fig. $3 a$ ), but the percentage of $\mathrm{Nte}^{-/-}$embryos decreased after E8, and no Nte ${ }^{-l}$ embryos were found after E11 (Fig. $3 a$ ). Nte $e^{-l-}$ embryos isolated at E9 had aberrant morphology and signs of resorption (Fig. 3b). This shows that Nte is essential for embryonic survival beyond E8. The cause of lethality is not apparent, but it may be due to defective closing of the neural tube. In contrast, the $\mathrm{Nte}^{+/-}$mice were viable and fertile.

\section{Nte expression patterns}

We stained $\mathrm{Nt} e^{+/-}$mice at various ages for $\beta$-galactosidase activity to identify the normal patterns of Nte expression (Fig. 4). We observed high levels of expression in restricted patterns throughout the $\mathrm{Nte}^{+/-}$adult mice and embryos. In particular, $\mathrm{Nte} \mathrm{t}^{+-}$ embryos at E13.5 showed strong expression in the cells of the

Fig. $3 \mathrm{Nte}^{-/-}$mice are not viable beyond E8. a, Changes in the genotype ratios (percentage distribution) during embryonic development showed that Nte is essential for embryo survival. +/+ indicates wild-type, +/- indicates heterozygote and $-I-$ indicates homozygote with respect to the mutated Nte allele. Total number of embryos were $n=31$ at E8, $n=22$ at E9, $n=36$ at E10, $n=24$ at E11 and $n=144$ post-natal (PN). At E8 the embryo distribution was close to the expected genotype ratio of $50 \%$ heterozygotes (blue line), $25 \%$ wild-type and $25 \%$ homozygotes (red line). But the percentage of $\mathrm{Nte}^{-/-}$embryos decreased through development and no $\mathrm{Nt}^{-/-}$mice were observed postnatally. $\boldsymbol{b}$, Photomicrographs of embryos isolated at E11, E10 and E9 with corresponding genotypes. E9 Nte ${ }^{-/-}$embryos seemed developmentally delayed and runted compared with age-matched $\mathrm{Nte}^{+/-}$and wild-type embryos. Nte ${ }^{-/-}$embryos at E10 and E11 seemed arrested in development, were much smaller than wildtype and $\mathrm{Nte}^{+/-}$embryos and showed signs of resorption. These observations suggest that $\mathrm{Nte}^{-/-}$embryos do not develop properly beyond E8. 
developing lens (Fig. 4b-f) and along the developing spinal cord (Fig. $4 a, b, g-j$ ). In the adult, expression was particularly robust in the Leydig cells of the testes, although faint staining was also observed in the testes of wild-type mice (Fig. $4 k-n$ ). Expression was observed throughout the brain, particularly in the cortex, in the Purkinje cells of the cerebellum (Fig. $40-v$ ) and in the hippocampus (Fig. $4 s-v$ ). These expression patterns are in agreement with northern-blot analysis (data not shown) and previous in situ hybridization experiments ${ }^{15}$.

\section{$\mathrm{Nte}^{+/-}$mice have lower activity of Nte but not acetylcholinesterase}

To determine the effect of disrupting one allele of Nte, we assessed the activities of Nte and acetylcholinesterase. We observed approximately $40 \%$ lower enzymatic activity of Nte in the brain in $\mathrm{Nte}^{+/}$ mice compared with wild-type mice (Fig. 5a; $P<0.0001$ ). A somewhat smaller reduction in Nte activity was observed in testes (Fig. $5 a$ ). This effect was specific to Nte, as there was no difference in the level of acetylcholinesterase activity between wild-type and $\mathrm{Nte}^{+/-}$mice (Fig. $5 a$ ). The level of Nte protein was also lower in the brain, testes and kidney (Fig. $5 b$ ) but not in the liver (data not shown), as determined by immunoprecipitation

Fig. 4 Nte is highly expressed in developing spinal cord and lens and in specific regions of the adult brain and testes. $\boldsymbol{a}, \boldsymbol{b}$, Whole-mount and sagittal sections of E13.5 embryos after $\beta$ galactosidase staining showed expression of $\mathrm{Nte}$ in the developing spinal cord (black arrow) and eye (white arrowhead). A wild-type embryo is shown on the left side of each panel and an $\mathrm{Nte}^{+/-}$embryo is on the right side of each panel. $c-f$, Thin sections of eye $(40 \mu \mathrm{m})$ confirmed that high levels of Nte expression were present in the developing lens. Sections of eyes from an $\mathrm{Nte}^{+-}$ embryo $(d, f)$ and a wild-type embryo $(c, e)$ were stained with X-gal. $\boldsymbol{g}$-j, Sections $(40 \mu \mathrm{m})$ of E13.5 embryos stained by Nissl $(g, i)$ or X-gal $(h, j)$, highlighting Nte expression in the developing spinal cord (arrows). $\boldsymbol{k}-\boldsymbol{n}$, Nte was abundantly expressed in the Leydig cells, as shown by X-gal staining of testes from an $\mathrm{Nte}^{+/-}$mouse (I). Low levels of non-specific X-gal staining were visible in testes from a wildtype mouse ( $n$ ). Nissl staining of testes is shown in $k$ and $m$. o- $r$, Nte was highly expressed in the Purkinje cell layer of the cerebellum as shown by $\mathrm{X}$-gal staining $(p, r)$. Cerebellar morphology can be observed in adjacent Nissl-stained sections $(0, q)$. $s-\boldsymbol{v}$, Horizontal sections showed that Nte seemed to be neuronal and was expressed in restricted regions throughout the brain, as indicated by $\mathrm{X}$-gal staining in brains from $\mathrm{Nte}^{+/}$ mice $(t)$ and wild-type mice $(v)$. In particular, expression was observed in the cortex and CA1-CA3 of hippocampus but not in the striatum or dentate gyrus (insets). Nissl sections show neuronal morphology $(s, u)$. and western blotting with a mouse-specific Nte antibody (Fig. 5b). Although the $\mathrm{Nte}^{+/-}$mice had lower Nte protein levels and activity, there were no signs of neuropathy or other obvious pathology.

\section{$\mathrm{Nte}^{+/-}$mice are more sensitive to organophosphate- induced toxicity}

To ascertain the role of Nte in organophosphate-induced toxicity, we tested whether $\mathrm{Nte}^{+/-}$mice were more or less sensitive than wild-type mice to an organophosphate compound that causes delayed toxicity. $\mathrm{Nte}^{+/-}$mice and wild-type littermates were injected intraperitoneally with 6 and $10 \mathrm{mg}$ EOPF per $\mathrm{kg}$ of body weight. The high potency of EOPF as an Nte inhibitor in vitro in mouse and hen brain $\left(\mathrm{IC}_{50}\right.$ values of $\left.0.02-0.04 \mathrm{nM}\right)$ and in vivo in mouse brain ( $85 \%$ inhibition at $5 \mathrm{mg}$ per $\mathrm{kg}$ body weight; refs. 19,25 ) makes it an optimal compound for studies in mice. We
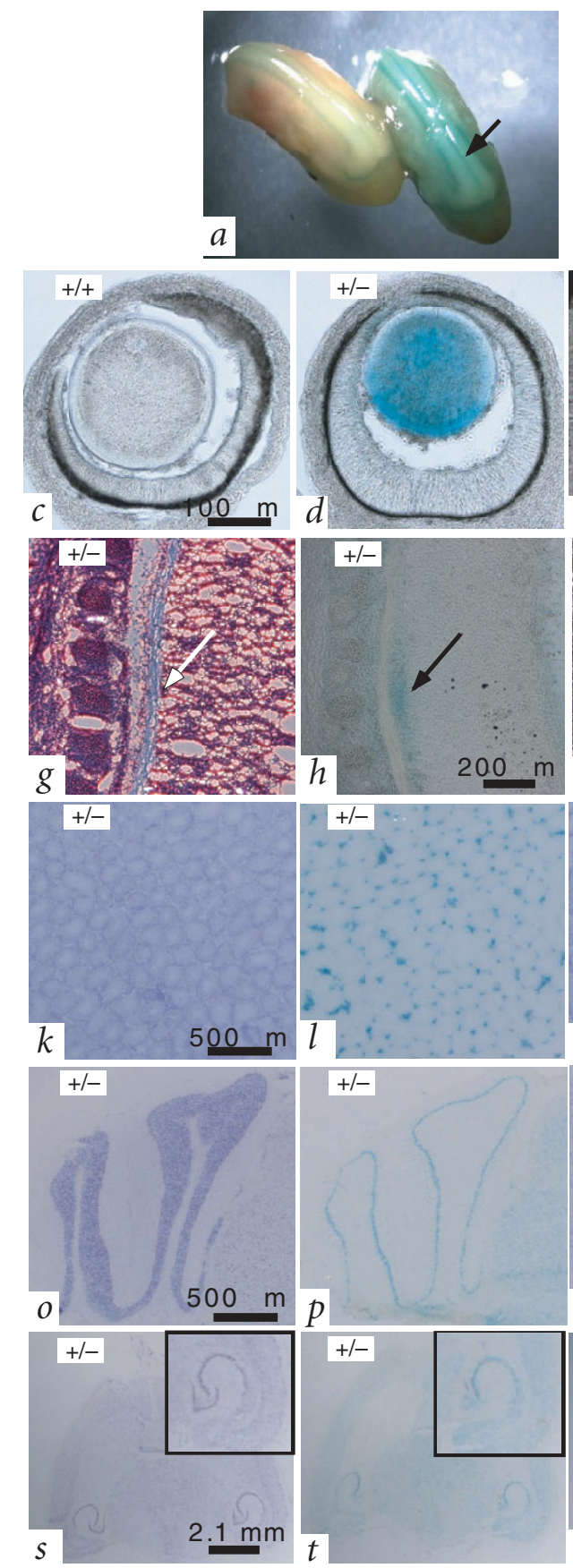
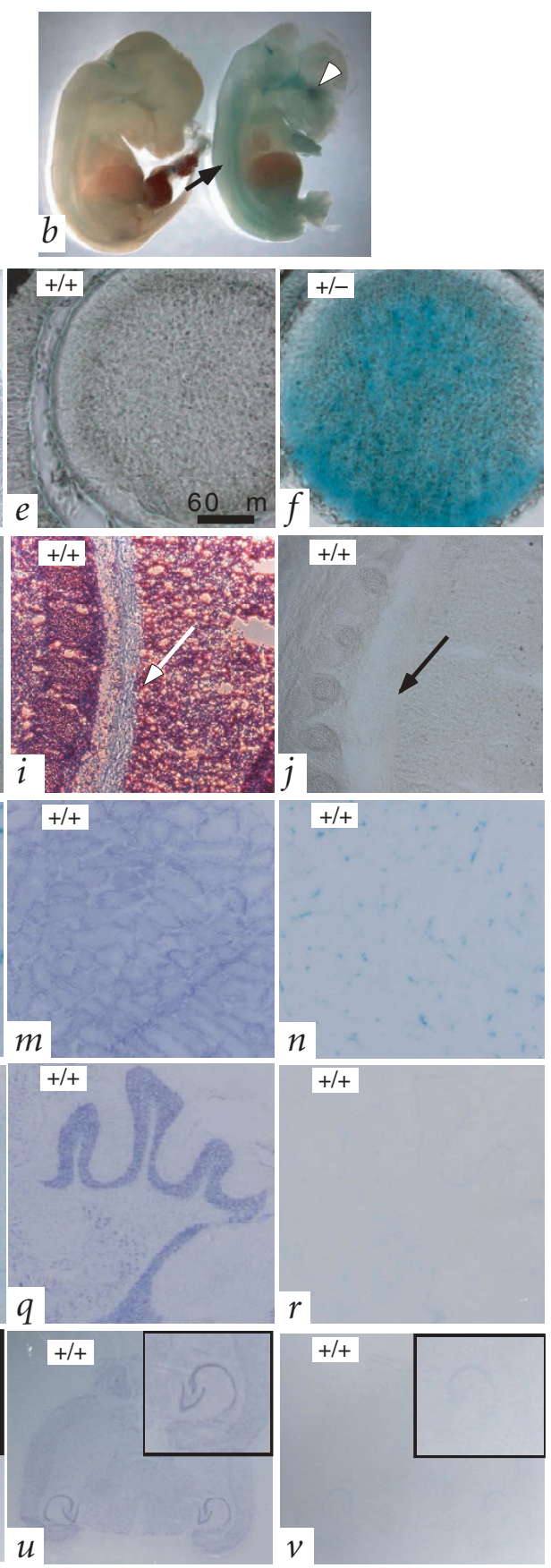
monitored clinical signs of mice treated with organophosphates and assessed mortality by Kaplan-Meier plot. The $\mathrm{Nte}^{+/-}$mice had significantly higher mortality rates at both 6 and $10 \mathrm{mg}$ EOPF per $\mathrm{kg}$ body weight (Fig. $6 a, b)$. Responses to EOPF included tearing, diminished movement and seizures. For comparison, no mortality or neuropathological effects were observed in either wild-type or $\mathrm{Nt}^{+/-}$mice treated with $1 \mathrm{mg}$ EOPF per $\mathrm{kg}$ body weight. These results indicate that EOPF acts through direct inhibition of Nte activity and that the loss of Nte function leads to EOPF-induced toxicity.

\section{Moderate reduction of Nte activity leads to greater motor activity}

We measured several aspects of neurological function in wildtype and $\mathrm{Nte}^{+/-}$mice in the presence and absence of a low dose of EOPF ( $1 \mathrm{mg}$ per $\mathrm{kg}$ body weight). In baseline studies without EOPF there were no significant differences between $\mathrm{Nte} \mathrm{e}^{+/-}$and wild-type mice in learning and memory, assayed by passive avoidance and audible conditioning experiments or response to a novel environment in standard open-field testing (data not shown). Based on these short-term measurements, $\mathrm{Nte}^{+/-}$mice showed no gross deficiencies in learning and memory or anxiety. Because of possible subtle latent effects, however, we extended our studies to examine long-term behavioral differences. We measured the locomotor activity of wild-type and $\mathrm{Nte}^{+/-}$mice (with or without exposure to $1 \mathrm{mg}$ EOPF per $\mathrm{kg}$ body weight) by open-field chamber testing after three days of acclimatization to the chambers. We recorded the total distance

$a$

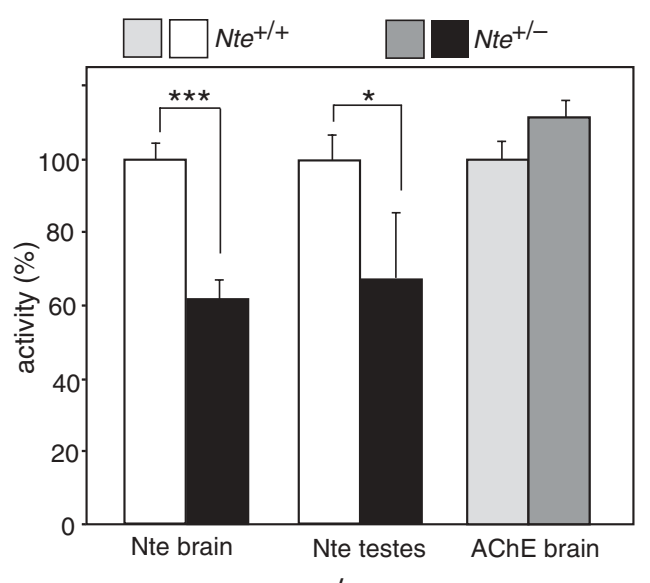

$b$

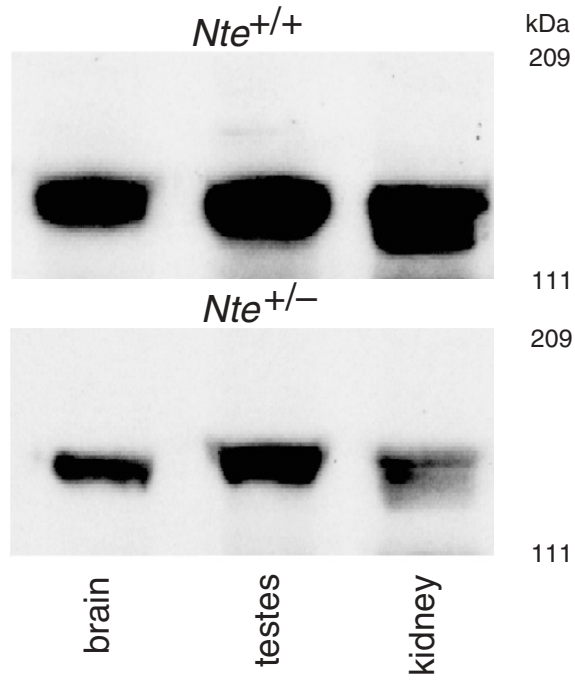

traveled and vertical counts over a ten-day interval. $\mathrm{Nte} e^{+/-}$mice showed higher baseline levels of motor activity than did wildtype mice, in terms of both distance traveled and vertical rearing (Fig. $6 c, d$ ). This is consistent with a phenotype of hyperactivity.

Because it was unclear whether this was due to lower Nte activity or developmental effects, we exposed the mice to $1 \mathrm{mg}$ EOPF per kg body weight and recorded their locomotor activity. After exposure to EOPF, the $\mathrm{Nte}^{+/-}$mice had markedly lower activity levels (Fig. $6 c, d$ ), probably owing to further reduction in their already-compromised Nte levels. The wild-type mice dosed with $1 \mathrm{mg}$ EOPF per kg body weight had significantly greater locomotor activity in terms of both total distance traveled (Fig. $6 c$ ) and vertical rearing (Fig. $6 d$ ). The difference in activity was almost identical to that seen at baseline (before exposure to EOPF) in the $N t e^{+/-}$mice. Histological examination of spinal cord and brain samples from wild-type and $\mathrm{Nt} e^{+/-}$mice at baseline and after treatment with $1 \mathrm{mg}$ EOPF per kg body weight did not show any marked differences (data not shown). Taken together, these findings indicate that even partial inhibition of NTE activity (either chemically or genetically) leads to an abnormal neurological phenotype in mammals.

\section{Discussion}

It has been proposed that long-term repeated exposure to NTE inhibitors can lead to permanent chronic neuropsychopathological disease affecting behavior as well as cognitive and visual functions (ref. 1; for review see ref. 26). It has been further postulated that neurotoxic chemical combinations, such as pesticides and an insect repellent, may cause more severe brain dysfunction ${ }^{3,27}$. Additional evidence suggests that pesticide exposure may lead to neurobehavioral effects such as attention-deficit hyperactivity disorder (refs. 28,29). Several important questions have yet to be answered in establishing the precise role of NTE and the consequences of its inhibition in mammals. Most importantly, the physiologic function of NTE is unknown. The generation of mice with Nte haploinsufficiency confirms that inhibition of Nte activity, either genetically or chemically, leads to neurological effects.

NTE maps to human chromosome 19p13.3. Two human disease loci have been reported in this region, although neither is associated with disruption of NTE (see Online Mendelian Inheritance in Man). The mutation responsible for Weill-Marchesani syndrome at 19p13.3-13.2 mapped to the WMS gene. The human mucolipidosis IV mutation at 19p13.3-13.2 mapped to the gene encoding mucolipin I.

We also identified a second member of the family, NTE-R1, on human chromosome 9 and mouse chromosome 2. The function of NTE-R1 is unknown, although it may be related to esterase activities in different fractions of hen brain and sciatic nerves $^{30-32}$. Our results clearly show that Nte-R1 cannot substitute for Nte during embryonic development and that it is not responsible for the effects of EOPF. The predicted NTE-R1 protein has a catalytic domain sharing homology with the NEST/patatin domain of NTE, but NTE-R1 functional esterase activity and substrate interactions have not yet been defined.

Fig. $5 \mathrm{Nte}^{+/-}$mice had lower activity of Nte but not acetylcholinesterase. a, $\mathrm{Nte}^{+/-}$mice had approximately $40 \%(P<0.0001)$ less activity of Nte in brain (solid black bar \pm s.e.m., $n=22)$ and $33 \%(P<0.05)$ less in testes (solid black bar \pm s.e.m., $n=6$ ) compared with wild-type littermates (solid white bars \pm s.e.m.). In contrast, activity of acetylcholinesterase (AChE) was not significantly different between $\mathrm{Nte}^{+-}$mice (hatched black bar \pm s.e.m.) and wild-type littermates (hatched white bar \pm s.e.m., $n=22$ ). $\boldsymbol{b}$, Nte protein levels in brain, testes and kidney were lower in $\mathrm{Nte}^{+--}$versus wild-type mice as analyzed by immunoprecipitation with a mouse Nte-specific antibody. A band predicted to be roughly $145 \mathrm{kDa}$ in size was observed. 
Fig. $6 \mathrm{Nte}^{+/-}$mice were more sensitive to organophosphate toxicity and showed greater motor activity. $\boldsymbol{a}, \boldsymbol{b}$, Kaplan-Meier plots show that $\mathrm{Nte}^{+-}$mice (solid line) had significantly higher mortality rates compared with wild-type littermates (dashed line) after exposure to EOPF. Significantly higher sensitivity was observed after treatment with $6 \mathrm{mg}$ EOPF per $\mathrm{kg}$ body weight $(\mathrm{mg} / \mathrm{kg})$ for $\mathrm{Nte} \mathrm{t}^{+/}$mice $(n=$ 25) compared with wild-type mice ( $n=$ 17; $P<0.0065 ;$ a). Similar results were seen after treatment with $10 \mathrm{mg}$ EOPF per kg body weight in $\mathrm{Nte}^{+/-}$mice $(n=29)$ compared with wild-type mice $(n=41 ; P<$ $0.0001 ; b) . c, d$, Motor activity in an openfield chamber as both distanced traveled (c) and vertical counts (d) was recorded at baseline and after challenging with EOPF (1 mg per kg body weight). The $x$ axis represents genotype and EOPF exposure and the $y$ axis indicates motor activity Baseline motor activity (before exposure to EOPF; pre-dose) was higher in $\mathrm{Nte}^{+/-}$ mice (black bars \pm s.e.m., $n=14$ ) com pared with wild-type mice (white bars \pm s.e.m., $n=18$ ). After treatment with EOPF motor activity was higher in wild-type mice (white bars \pm s.e.m., $n=4$ ) and lower in $\mathrm{Nte}^{+/-}$mice (black bars \pm s.e.m., $n=5$ ). Significance was determined for all results presented using an unpaired $t$ test: ${ }^{*}, P<0.05 ; * *, P<0.01 ; * * *, P<0.001$. the Purkinje cells of the cerebellum and in the spinal cord, but also in the Leydig cells of the testes and in the developing lens. Therefore, there probably are organismal effects of reducing NTE activity outside of the nervous system. Notably, the organophosphate phosphamidon leads to toxic effects in Leydig cells and clear cells of the cauda in the testes of rats ${ }^{33}$, and environmental exposure to diazinon leads to retinal cell necrosis in teleosts ${ }^{34}$. Our results show that Nte is essential for embryonic survival, pointing to important consequences of inhibition of NTE in the developing vertebrate embryo and highlighting the key roles for NTE in mammalian embryogenesis and development.

A seminal observation is that moderate reduction in Nte activity, by either reducing the amount of Nte protein through genetics or using a potent Nte inhibitor, leads to hyperactivity. The mechanism of this possible cause-and-effect relationship has yet to be determined. The biochemical function of NTE is unknown, but considerable progress has been made recently in defining its physiological substrate. The catalytic domain of NTE alters membrane conductivity in liposome preparations, and neuropathic organophosphates change this response in vitro ${ }^{35}$. Human NTE hydrolyzes membrane associated lipids, pointing to a role for NTE in lipid metabolism or signaling ${ }^{36}$. NTE contains several cNMP-binding domains commonly found in intracellular signaling factors and cyclic nucleotide gated ion channels ${ }^{10,36,37}$ There is a good homology of the key residues around the active sites of NTE and calcium-independent phospholipase A2 (ref. 36). In this context, it is important to note that attention-deficit hyperactivity disorder has been linked to disorders involving lipid metabolism ${ }^{38}$, pesticide exposure ${ }^{28,29}$ and changes in ionchannel conductance in rat models ${ }^{39}$. Taken together, this evidence suggests that in mammals, the catalytic activity of NTE is linked to the control of motor activity and that the inhibition of NTE in humans may contribute to some hyperactivity disorders.

The neuropathies in hens arising from organophosphate inhibition of NTE cause wallerian axonal degeneration and show vacuolation and neuronal swelling over a period of 1-3 weeks ${ }^{40,41}$. Although the hen is the preferred model for OPIDN studies, there is growing evidence that this model may not properly estimate the risk of organophosphate exposure in humans and other mammals ${ }^{42}$ and does not address short- and mediumterm toxicities of organophosphates or non-neuropathy longterm sequelae. It is important that mice do not give a typical OPIDN response ${ }^{21}$ and the mechanistic relationship between OPIDN in hens and delayed toxicity in mice has not yet been determined. In our study, no signs of neuropathy were observed in the $\mathrm{Nte} \mathrm{t}^{+-}$mice that had $40 \%$ reduction in Nte activity, consistent with the 70-90\% inhibition of Nte activity normally associated with organophosphate-induced delayed toxicity in wild-type mice ${ }^{19,25}$ and OPIDN in hens ${ }^{11-13}$. But the $\mathrm{Nte}^{+/-}$mice were more sensitive to organophosphate-induced subacute toxicity, manifested by greater mortality, making it difficult to assess the long-term effects of exposure to higher levels of EOPF.

Neuropathology due to NTE inhibition by organophosphates is proposed to result from a toxic gain of function on aging of phosphorylated NTE and not merely from a loss of normal enzymatic activity ${ }^{10,23}$. Such a toxic gain of function is implicated in several neurodegenerative diseases, including familial amyotrophic lateral sclerosis ${ }^{43}$, and in CAG triplet diseases, such as Huntington disease ${ }^{44}$. But our results show that even partial inhibition of Nte results in a clear neurobehavioral phenotype of hyperactivity. The experiments with Nte-deficient mice show that it is the reduction in Nte activity and not the generation of aged Nte that is responsible for the phenotype. The reduced level of Nte protein in the $\mathrm{Nte} e^{+-}$mice makes them more susceptible to the toxic effects of EOPF, establishing that it is a loss rather than a gain of function that results in toxicity. In addition, this study shows that chemical inhibition of Nte by EOPF mimics the phenotype caused by partial loss of Nte through genetic haploinsufficiency. Therefore, we clearly show for the first time that organophosphates that can cause neurological effects act through inhibition of Nte (without the requirement for aging or 
gain of function) and this inhibition may be detrimental to the nervous system of mammals. The Nte-haploinsufficient mouse model will help define the relationship between NTE, delayed toxicity and OPIDN and may also be a useful model for understanding how nerve gas poisons and pesticides act individually or in combination to cause neurological dysfunction in man.

More than half a century of research with organophosphate toxicants has led to the discovery of several toxicological manifestations not attributable to acetylcholinesterase inhibition ${ }^{1}$, including Gulf War syndrome $e^{1,6,26,45}$. NTE inhibition may be involved in some of these secondary events. The generation of mice lacking Nte is an important step in defining its function in mammals. The use of Nte-deficient mice may hasten the development of therapies for organophosphate-induced delayed toxicities and neuropathies. These findings show that Nte is highly expressed in the nervous system, that Nte is essential for embryonic development and survival and that mice lacking one allele of Nte are viable and fertile but have lower enzyme activity in the brain and are more susceptible to EOPF toxicity. Furthermore, a moderate reduction in Nte activity, either genetically or by administration of EOPF, is sufficient to produce hyperactivity. These observations provide biological evidence of the need to redefine the relationship between NTE, delayed toxicity and OPIDN in mammals. Clearly, Nte is an essential gene for development and for regulating motor activity.

\section{Methods}

Gene targeting and production of Nte-disrupted mice. We used a mouse EST to design PCR primers to screen a 129S6 mouse genomic BAC library (Incyte Genomics, Down-to-the-well) according to manufacturer's directions. PCR yielded a genomic fragment of $1122 \mathrm{bp}$ and a cDNA fragment of 169 bp. Primer sequences are available on request. The BAC clone 59B13 was identified and characterized (Fig. 1c). We obtained and characterized the human BAC 48M5 (Incyte Genomics). We used a 160-bp probe to screen a liver cDNA library (Lifetech) and identified a 1659-bp alternatively spliced clone in addition to the full-length Nte (Fig. 1c; ref. 15). Isolation of a $5-\mathrm{kb}$ BamHI-BstEII fragment containing exons $1-3$ and a $5-\mathrm{kb}$ SmaI-EcoRI fragment containing exons 10-15 enabled us to generate the targeting construct. We added a Bam HI linker to the BstEII site in exon 3 and fused this in-frame to the lac $Z$ gene. The Nte-lacZ fragment was inserted into the pPNT/PGKNeo vector ${ }^{46}$ at an XhoI site, and the SmaI-EcoRI fragment was inserted as a blunt fragment into the KpnI site of pPNT/PGKNeo adjacent to the thymidine kinase (HSV-TK) gene (Fig. $2 a, b)$. We linearized and transfected the vector into 129S6 ES cells as previ ously described ${ }^{47}$. Using an internal probe and BstEII digestion for Southern-blot analysis, we screened 114 G418-resistant and gancyclovir-sensitive ES cell clones (Fig. 2a). We identified nine positive clones. Extensive PCR, restriction-enzyme digestion and Southern-blot analyses with both internal and external primers and probes ensured selection of ES clones containing the correctly targeted locus (data not shown). We microinjected targeted clones into C57BL/6J blastocysts using standard methods. Two clones yielded chimeras capable of high-level germline transmission of the disrupted Nte allele as determined by mating with C57BL/6J mice. We established two inbred 129S6 lines harboring the disrupted Nte allele from the independent ES clones. We carried out genotyping by Southern-blot analysis as described above (Fig. 2c) and by PCR using a forward primer for a site $5^{\prime}$ to the targeted region and reverse primers for a site in the targeted region and in the lac $Z$ gene (Fig. $2 d$ ). All animal procedures were carried out according to protocols approved by the Salk Institute for Biological Studies Animal Care and Use Committee.

FISH analysis. We prepared metaphase spreads for FISH on glass slides using standard protocols. We incubated cells in $0.1 \mathrm{mg} \mathrm{ml}^{-1}$ colcemid (GIBCO/BRL) for 30-60 min and then lysed them in $0.075 \mathrm{M} \mathrm{KCl}$. We fixed chromosomes in 3:1 methanol:acetic acid and dropped them onto glass slides. We generated probes for FISH using BAC clones containing the genes of interest. We generated labeled BAC probes using the BioProbe nick-translation kit (Sigma). The BAC DNA clones were labeled with
biotin-16-dUTP, digoxigenin-11-dUTP (Roche) or Spectrum OrangedUTP (Vysis). Nick-translated probe DNA (100 ng) was precipitated with $15 \mu \mathrm{g}$ mouse $\mathrm{C}_{\mathrm{o}} \mathrm{t}-1$ DNA (Gibco) and resuspended in $50 \%$ formamide, $10 \%$ dextran sulfate, $2 \times$ saline-sodium citrate buffer. The probe DNA was denatured $\left(10 \mathrm{~min}\right.$ at $\left.75^{\circ} \mathrm{C}\right)$ and metaphase spreads were pretreated with RNase A $\left(0.1 \mathrm{mg} \mathrm{ml}^{-1}\right.$ for $1 \mathrm{~h}$ at $\left.37^{\circ} \mathrm{C}\right)$ and pepsin $\left(0.1 \mathrm{mg} \mathrm{ml}^{-1}\right.$ for $10 \mathrm{~min}$ at $\left.37^{\circ} \mathrm{C}\right)$ and then fixed in formalin ( $1 \%$ for $10 \mathrm{~min}$ at room temperature). After 30 min preannealing of probe DNA, we hybridized it to metaphase spreads for $24 \mathrm{~h}$ at $37^{\circ} \mathrm{C}$ in a humidified box ${ }^{48}$. After hybridization, we detected indirectly labeled probes with either mouse antibody against digoxigenin followed by sheep antibody against mouse Cy5.5 or avidin-fluorescein isothiocyanate. FISH results were imaged and analyzed using QFISH software (Leica).

Staining for $\beta$-galactosidase activity. We obtained sections $(10 \mu \mathrm{m})$ of fresh frozen tissue samples from wild-type and $N t^{+/-}$mice, fixed them in GTS fixation solution and visualized activity of 5-bromo-4-chloro-3indolyl- $\beta$-D-galactoside (X-gal) using the X-gal Staining Assay Kit according to the manufacturer's directions (Gene Therapy Systems). We collected wild-type and $N t e^{+/-}$embryos into phosphate-buffered saline at $4{ }^{\circ} \mathrm{C}$. We fixed the embryos for $30 \mathrm{~min}$ in GTS fixation buffer and then incubated them three times for $10 \mathrm{~min}$ in a detergent rinse $(0.1 \mathrm{mM}$ phosphate buffer ( $\mathrm{pH} 7.3$ ), $2 \mathrm{mM} \mathrm{MgCl}, 0.01 \%$ sodium deoxycholate and $0.02 \%$ Nonidet $\mathrm{P}-40)$ before staining for X-gal activity as described above. We also prepared sections $(40 \mu \mathrm{m})$ of embryos stained with X-gal to enable additional visualization of the developing lens and spinal cord. Nissl staining was carried out according to a standard protocol.

Activities of Nte and acetylcholinesterase. We homogenized brain $(20 \%$ $\mathrm{w} / \mathrm{v})$ and testes $(10 \% \mathrm{w} / \mathrm{v})$ in $50 \mathrm{mM}$ Tris buffer ( $\mathrm{pH} 8.0)$ containing $0.2 \mathrm{mM}$ ethylenediamine tetraacetic acid (EDTA). Homogenates were centrifuged at $700 \mathrm{~g}$ for $10 \mathrm{~min}$, pellets discarded and supernatants used for Nte and acetylcholinesterase assays. NTE is considered to be that portion of the phenyl valerate-hydrolyzing activity that is insensitive to paraoxon $(40 \mu \mathrm{M})$ but sensitive to mipafox $(50 \mu \mathrm{M})$. We determined activity of mouse brain Nte at $37{ }^{\circ} \mathrm{C}$ by a modification of the procedure for hen brain $\mathrm{NTE}^{19,49}$. Homogenates $(50 \mu \mathrm{l}$ ) were diluted 20 -fold in Tris-EDTA buffer (as above; $950 \mu \mathrm{l})$. Paraoxon $(11 \mu \mathrm{g}, 40 \mu \mathrm{M}$ final concentration) was added in acetone $(10 \mu \mathrm{l})$ and mipafox $(0$ or $9.1 \mu \mathrm{g}, 50 \mu \mathrm{M}$ final concentration) was added in acetone $(10 \mu \mathrm{l})$. After $20 \mathrm{~min}$ incubation, phenyl valerate $(480 \mu \mathrm{g})$ was introduced in $0.03 \%$ Triton X-100 in Tris-EDTA buffer $(1 \mathrm{ml})$ and incubated for $15 \mathrm{~min}$. The reaction was stopped with $1 \%$ SDS and $0.025 \% 4$-aminoantipyrine in water $(1 \mathrm{ml})$. Adding $0.4 \%$ potassium ferricyanide in water $(0.5$ $\mathrm{ml}$ ) and waiting $5 \mathrm{~min}$ allowed colorimetric determination of Nte activity at $510 \mathrm{~nm} . \mathrm{A}_{510}$ values for wild-type brain $(n=14)$ were $1.02 \pm 0.08$ with paraoxon and $0.68 \pm 0.05$ with paraoxon plus mipafox, giving Nte activity of 0.34 (about $10 \%$ of the total phenyl valerate-hydrolyzing activity). Testes had more variable Nte activity assays with $\mathrm{A}_{510}$ values of $0.92 \pm 0.10,0.78 \pm$ 0.12 and 0.15 , respectively $(n=13)$. We assayed acetylcholinesterase activity with acetylthiocholine $(0.6 \mathrm{mM})$ and 5,5'-dithio-bis(2-nitrobenzoic acid) (1 $\mathrm{mM})$ in $100 \mathrm{mM}$ phosphate $(\mathrm{pH} 7.4,1.1 \mathrm{ml})$ to which the undiluted homogenate $(20 \mu \mathrm{l})$ was added and the absorbance increase continuously monitored over a period of $10 \mathrm{~min}$ at $412 \mathrm{~nm}^{50}$.

Immunoprecipitation and determination of protein expression. We generated column-purified mouse Nte-specific antibodies against a peptide corresponding to amino acids 34-52 (RLRVQKTPAPEGPRYRFRK; Alpha Diagnostics). We homogenized tissue in Nonidet P-40 lysis buffer $(20 \mathrm{mM}$ Tris- $\mathrm{HCl}, \mathrm{pH}$ 8.0, $137 \mathrm{mM} \mathrm{NaCl}, 10 \%$ glycerol, 1\% Nonidet P-40, $1 \mathrm{mM}$ EDTA, protease inhibitors (aprotinin + leupeptin), $1 \mathrm{mM}$ phenylmethylsulfonyl fluoride) at $4{ }^{\circ} \mathrm{C}$. The samples were then centrifuged and the supernatant was removed and stored. The protein supernatant $(1 \mathrm{mg})$ was pre-cleared with Protein-A Sepharose slurry for $30 \mathrm{~min}$, then removed and incubated with the Protein-A Sepharose for $2 \mathrm{~h}$ with the mouse Nte antibody (1:125 concentration). We removed the Protein-A Sepharose beads and washed samples, and protein was liberated on incubation with SDS buffer at $70{ }^{\circ} \mathrm{C}$ for $10 \mathrm{~min}$. The supernatant was then run on a Tris-acetate gel, blotted and visualized with a secondary horseradish peroxidase-conjugate antibody and detected using the ECL kit (Amersham). Extra bands were observed in addition to the one corresponding to Nte, precluding use of this antibody for immunohistochemistry. 
EOPF administration and toxicity studies. We dissolved EOPF $(99 \%$ pure; synthesized in G.B.Q. and J.E.C.'s laboratory) in dimethylsulfoxide immediately before administration to the test mice. Mice were weighed immediately before EOPF treatment and were given a single intraperitoneal injection of EOPF corresponding to 1, 6 or $10 \mathrm{mg}$ per $\mathrm{kg}$ body weight. Control mice received an injection of an equivalent volume of dimethylsulfoxide. We monitored the activity, appearance and mortality of the mice twice daily and measured weight daily during the course of the experiments.

Behavioral testing and assessment of locomotor activity. We carried out behavioral experiments measuring distance traveled and vertical counts using Open Field Test Chambers (Med Associates model ENV-515) of clear Plexiglas $(43.2 \mathrm{~cm} \times 43.2 \mathrm{~cm} \times 30.5 \mathrm{~cm})$ to monitor open-field activity in the light phase of the test mice's light/dark cycle. We took the mice from the home cage, placed them into the behavioral testing chamber for $15 \mathrm{~min}$ and then removed them directly back to the home cage. The open-field test chambers measured ambulatory distance, ambulatory counts, vertical counts and the time of each activity by monitoring light beams broken during the movement. We analyzed the data using Activity Monitor version 4.0 (Med Associates). We carried out standard open-field measures as described ${ }^{46}$. For total locomotor activity assays, we tested mice once per day over consecutive days. For baseline measurements, we tested 18 wild-type ( 9 female and 9 male) and $14 \mathrm{Nte} \mathrm{t}^{+/-}$( 6 female and 8 male) mice. We tested 4 wild-type ( 3 female and 1 male) and $5 \mathrm{Nte}^{+/-}$(4 female and 1 male) mice that were treated with $1 \mathrm{mg}$ EOPF per kg body weight. The testing trials lasted over a 12-d period. The first $2 \mathrm{~d}$ were for acclimation and thus were omitted from the total locomotor activity analysis. The data from the first $2 \mathrm{~d}$ were analyzed separately for behavioral differences at 5, 10 and $15 \mathrm{~min}$ intervals but showed no difference between the groups. We averaged the measurements of all mice of each group and activity over $10 \mathrm{~d}$ (after acclimation) to compare wild-type with $\mathrm{Nte}^{+/-}$mice.

We carried out passive avoidance tests for $2 \mathrm{~d}$ using a chamber (Med Associates, ENV-010MC) with two rooms, one brightly lit and one dark, separated by a small door. On day 1 , we placed mice on the lighted side of the chamber for $10 \mathrm{~s}$, opened the door to enable them to enter the dark side of the chamber, shut behind them and applied a foot shock for $3 \mathrm{~s}$. We then removed the mice from the chamber and returned them to their home cage. On day 2, we again placed mice on the lighted side of the chamber and opened the door after a 10-s delay and then measured latency to enter. After the mice entered the dark side of the cage, the door was again shut but no shock was applied. We then returned the mice to their home cage. We applied foot shocks of $0.5 \mathrm{~mA}$ (wild-type, $n=29$; $\mathrm{Ne}^{+/-}, n=31$ ) and $0.75 \mathrm{~mA}$ (wild-type, $n=17 ; \mathrm{Nte}^{+/-}, n=13$ ) in separate experiments.

We carried out fear conditioning according to standard protocols. The test measures freezing responses in mice over a 7-d testing period. We used a shocking chamber (Med Associates, ENV-010MC) and a clear plexiglass chamber to test the mice. On day 1, we placed mice in the shocking chamber. After $160 \mathrm{~s}$, three tone-shock pairs were delivered at intervals of $1 \mathrm{~min}$ A tone-shock pair consists of $20 \mathrm{~s}$ of white noise followed by a 1-s $0.75-\mathrm{mA}$ foot shock. After the tone-shocks, we kept the mice in the chamber for $60 \mathrm{~s}$ before returning them to the home cage. On day 2 , we placed mice back in the shocking chamber 6 min and measured freezing to the context. On day 3 , we put the mice into the clear plexiglass chamber. After $160 \mathrm{~s}$, we delivered three 20-s tones identical to those in the tone-shock pairs delivered on day 1 . The trial lasted for a total of $6 \mathrm{~min}$. Freezing before the tones was considered a response to an unconditioned context. Freezing after the tones were delivered was considered a response to the tone. We conducted context extinction trials on days 4-7. These trials consisted of placing the mice back into the shocking chamber for 6 min each day. Freezing, which was measured as complete lack of movement in the body and minimal movement of the head, was timed using a stopwatch. We tested wild-type $(n=4)$ and $N t e^{+/-}(n=4)$ mice.

Statistical analysis. We generated Kaplan-Meier survival plots to analyze the mortality data and measured significance using the BreslowGehan-Wilcoxon test, the Tarone-Ware test, the Peto-Peto-Wilcoxon test and the Harrington-Fleming test $(\mathrm{rho}=0.5)$. Significant differences in Nte and acetylcholinesterase activities, behavioral distance traveled and vertical counts were determined using an unpaired $t$-test. One asterisk represents $P<0.05$; two asterisks, $P<0.01$; and three asterisks, $P<0.001$.
Sequence and bioinformatics information. Analyses of the Celera Discovery System and NCBI databases were in agreement with our cytogenetic and sequence data. We searched the NCBI public databases (including LocusLink, GenBank, Online Mendelian Inheritance in Man, Mouse-Human Homology Maps and Unigene) and the Mouse Genome Database. In addition, we generated data using the Celera Discovery System and Celera's associated databases.

URLs. NCBI, http://www.ncbi.nlm.nih.gov; Mouse Genome Database, http://www.informatics.jax.org.

Accession numbers. The accession numbers for nucleic acid sequences described in this study are as follows: mouse Nte 5' EST, NCBI W18687; the NTE-R1 locus from mouse, NCBI NM_146251 (partial) and Celera mCG20285; rat, NCBI NM_144738 (partial); and human, NCBI NM_152286 (partial) and Celera hCG1811431.

\section{Acknowledgments}

The authors thank L. Garrett, J. Cheng, Y. Dayn and K-F. Lee for assistance in generating transgenic mice, D. Wangsa for assistance with cytogenetic analyses, $R$. Helton for animal husbandry, E. Annas for technical support and B. Cravatt and S. Heinemann for comments and experimental advice. This work was supported by the Canadian Institutes of Health Research (C.J.W.), the US National Institute of Environmental Health Sciences, the US National Institutes of Health (J.E.C), the Department of Defense (US Army Medical Research and Material Command) and the Frederick B. Rentschler Endowed Chair (C.B.).

\section{Competing interests statement}

The authors declare that they have no competing financial interests.

Received 10 December 2002; accepted 21 February 2003.

1. Karczmar, A.G. Acute and long lasting central actions of organophosphorus agents. Fundam. Appl. Toxicol. 4, S1-17 (1984).

2. Solberg, Y. \& Belkin, M. The role of excitotoxicity in organophosphorous nerve agents central poisoning. Trends Pharmacol. Sci. 18, 183-185 (1997).

3. Haley, R.W. \& Kurt, T.L. Self-reported exposure to neurotoxic chemical combinations in the Gulf War. A cross-sectional epidemiologic study. JAMA 277, 231-237 (1997).

4. Enserink, M. Gulf War illness: the battle continues. Science 291, 812-817 (2001).

5. Hitt, E. New investigations into Gulf War syndrome. Nat. Med. 8, 198 (2002).

6. Haley, R.W. et al. Evaluation of neurologic function in Gulf War veterans. A blinded case-control study. JAMA 277, 223-230 (1997).

7. Lotti, M. Low-level exposures to organophosphorus esters and peripheral nerve function. Muscle Nerve 25, 492-504 (2002).

8. Ray, D.E. \& Richards, P.G. The potential for toxic effects of chronic, low-dose exposure to organophosphates. Toxicol. Lett. 120, 343-351 (2001).

9. Jamal, G.A. Gulf War syndrome-a model for the complexity of biological and environmental interaction with human health. Adverse Drug React. Toxicol. Rev. 17, 1-17 (1998).

10. Glynn, P. Neuropathy target esterase. Biochem. J. 344, 625-631 (1999).

11. Johnson, M.K. The primary biochemical lesion leading to the delayed neurotoxic effects of some organophosphorus esters. J. Neurochem. 23, 785-789 (1974).

12. Glynn, P. Neural development and neurodegeneration: two faces of neuropathy target esterase. Prog. Neurobiol. 61, 61-74 (2000).

13. Johnson, M.K. \& Glynn, P. Neuropathy target esterase. in Handbook of Pesticide Toxicology, Vol. 2 (ed. Krieger, R.I.) 953-965 (Academic Press, San Diego, 2001).

14. Lush, M.J., Li, Y., Read, D.J., Willis, A.C. \& Glynn, P. Neuropathy target esterase and a homologous Drosophila neurodegeneration-associated mutant protein contain a novel domain conserved from bacteria to man. Biochem. J. 332, 1-4 (1998).

15. Moser, M. et al. Cloning and expression of the murine sws/NTE gene. Mech. Dev. 90, 279-282 (2000)

16. Kretzschmar, D. Hasan, G., Sharma, S., Heisenberg, M. \& Benzer, S. The swiss cheese mutant causes glial hyperwrapping and brain degeneration in Drosophila. J. Neurosci. 17, 7425-7432 (1997).

17. Veronesi, B., Ehrich, M., Blusztajn, J.K., Oortgiesen, M. \& Durham, H. Cell culture models of interspecies selectivity to organophosphorous insecticides. Neurotoxicology 18, 283-297 (1997).

18. Husain, K., Vijayaraghavan, R., Pant, S.C., Raza, S.K. \& Pandey, K.S. Delayed neurotoxic effect of sarin in mice after repeated inhalation exposure. J. Appl. Toxicol. 13, 143-145 (1993).

19. Wu, S.Y. \& Casida, J.E. Subacute neurotoxicity induced in mice by potent organophosphorus neuropathy target esterase inhibitors. Toxicol. Appl. Pharmacol. 139, 195-202 (1996).

20. Meredith, C. \& Johnson, M.K. Neuropathy target esterase: rates of turnover in vivo following covalent inhibition with phenyl di-n-pentylphosphinate. J. Neurochem. 51, 1097-1101 (1988).

21. Ehrich, M. \& Jortner, B.S. Organophosphorous-induced delayed neuropathy. in Handbook of Pesticide Toxicology, Vol. 2 (ed. Krieger, R.I.) 987-1012 (Academic Press, San Diego, 2001)

22. Ehrich, M., Jortner, B.S. \& Padilla, S. Relationship of neuropathy target esterase inhibition to neuropathology and ataxia in hens given organophosphorus esters. Chem. Biol. Interact. 87, 431-437 (1993). 
23. Atkins, J. \& Glynn, P. Membrane association of and critical residues in the catalytic domain of human neuropathy target esterase. J. Biol. Chem. 275, 24477-24483 (2000).

24. Mignery, G.A., Pikaard, C.S. \& Park, W.D. Molecular characterization of the patatin multigene family of potato. Gene 62, 27-44 (1988).

25. Wu, S.Y. \& Casida, J.E. Ethyl octylphosphonofluoridate and analogs: optimized inhibitors of neuropathy target esterase. Chem. Res. Toxicol. 8, 1070-1075 (1995).

26. Jamal, G.A. Neurological syndromes of organophosphorus compounds. Adverse Drug React. Toxicol. Rev. 16, 133-170 (1997).

27. Wilson, B.W., Henderson, J.D., Coatney, E.M., Nieberg, P.S. \& Spencer, P.S. Actions of pyridostigmine and organophosphate agents on chick cells, mice, and chickens. Drug Chem. Toxicol. 25, 131-139 (2002).

28. Schettler, T. Toxic threats to neurologic development of children. Environ. Health Perspect. 109 Suppl 6, 813-816 (2001)

29. Hardell, L., Lindstrom, G. \& Van Bavel, B. Is DDT exposure during fetal period and breast-feeding associated with neurological impairment? Environ. Res. 88, 141-144 (2002).

30. Vilanova, E., Barril, J. \& Carrera, V. Biochemical properties and possible toxicological significance of various forms of NTE. Chem. Biol. Interact. 87, 369-381 (1993).

31. Escudero, M.A., Cespedes, M.V. \& Vilanova, E. Chromatographic discrimination of soluble neuropathy target esterase isoenzymes and related phenyl valerate esterases from chicken brain, spinal cord, and sciatic nerve. J. Neurochem. 68 esterases from chick

32. Tormo, N., Gimeno, J.R., Sogorb, M.A., Diaz-Alejo, N. \& Vilanova, E. Soluble and particulate organophosphorus neuropathy target esterase in brain and sciatic nerve of the hen, cat, rat, and chick. J. Neurochem. 61, 2164-2168 (1993).

33. Akbarsha, M.A. \& Sivasamy, P. Male reproductive toxicity of phosphamidon: histopathological changes in epididymis. Indian J. Exp. Biol. 36, 34-38 (1998).

34. Hamm, J.T., Wilson, B.W. \& Hinton, D.E. Organophosphate-induced acetylcholinesterase inhibition and embryonic retinal cell necrosis in vivo in the teleost (Oryzias latipes). Neurotoxicology 19, 853-869 (1998)

35. Forshaw, P.J., Atkins, J., Ray, D.E. \& Glynn, P. The catalytic domain of human neuropathy target esterase mediates an organophosphate-sensitive ionic conductance across liposome membranes. J. Neurochem. 79, 400-406 (2001).

36. van Tienhoven, M., Atkins, J., Li, Y. \& Glynn, P. Human neuropathy target esterase catalyses hydrolysis of membrane lipids. J. Biol. Chem. 277, 20942-20948 (2002).

37. Yau, K.W. Cyclic nucleotide-gated channels: an expanding new family of ion channels. Proc. Natl. Acad. Sci. USA 91, 3481-3483 (1994).

38. Burgess, J.R., Stevens, L., Zhang, W. \& Peck, L. Long-chain polyunsaturated fatty acids in children with attention-deficit hyperactivity disorder. Am. J. Clin. Nutr. 71, 327S-330S (2000).

39. Ishimatsu, M., Kidani, Y., Tsuda, A. \& Akasu, T. Effects of methylphenidate on the membrane potential and current in neurons of the rat locus coeruleus. J. Neurophysiol. 87, 1206-1212 (2002).

40. De Bleecker, J.L., De Reuck, J.L. \& Willems, J.L. Neurological aspects of organophosphate poisoning. Clin. Neurol. Neurosurg. 94, 93-103 (1992).

41. Randall, J.C., Yano, B.L. \& Richardson, R.J. Potentiation of organophosphorus compound-induced delayed neurotoxicity (OPIDN) in the central and peripheral nervous system of the adult hen: distribution of axonal lesions. J. Toxicol. Environ. Health 51, 571-590 (1997).

42. Moretto, A. \& Lotti, M. The relationship between isofenphos cholinergic toxicity and the development of polyneuropathy in hens and humans. Arch. Toxicol. 76, 367-375 (2002).

43. Casareno, R.L., Waggoner, D. \& Gitlin, J.D. The copper chaperone CCS directly interacts with copper/zinc superoxide dismutase. J. Biol. Chem. 273, 23625-23628 (1998).

44. Ross, C.A. et al. Polyglutamine pathogenesis. Philos. Trans. R. Soc. Lond. B Biol. Sci. 354, 1005-1011 (1999).

45. Cavanagh, J.B. Peripheral neuropathy caused by chemical agents. CRC Crit. Rev. Toxicol. 2, 365-417 (1973).

46. Barlow, C. et al. Atm-deficient mice: a paradigm of ataxia telangiectasia. Cel/ $\mathbf{8 6}$, 159-171 (1996).

47. Deng, C., Wynshaw-Boris, A., Zhou, F., Kuo, A. \& Leder, P. Fibroblast growth factor receptor 3 is a negative regulator of bone growth. Cell 84, 911-921 (1996).

48. Ried, T., Landes, G., Dackowski, W., Klinger, K. \& Ward, D.C. Multicolor fluorescence in situ hybridization for the simultaneous detection of probe sets for chromosomes 13,18, 21, X and $\mathrm{Y}$ in uncultured amniotic fluid cells. Hum. Mol. Genet. 1, 307-313 (1992).

49. Johnson, M.K. Improved assay of neurotoxic esterase for screening organophosphates for delayed neurotoxicity potential. Arch. Toxicol. 37, 113-115 (1977).

50. Ellman, G.L., Courtney, K.D., Andres Jr., V. \& Featherstone, R.M. A new and rapid colorimetric determination of acetylcholinesterase activity. Biochem. Pharmacol. 7, 88-95 (1961). 